\title{
Advanced simulations of optical transition and diffraction radiation
}

\author{
T. Aumeyr, ${ }^{1, *}$ M. G. Billing, ${ }^{2}$ L. M. Bobb, ${ }^{1,3}$ B. Bolzon, ${ }^{3,4}$ E. Bravin, ${ }^{3}$ \\ P. Karataev, ${ }^{1}$ K. Kruchinin, ${ }^{1}$ T. Lefevre, ${ }^{3}$ and S. Mazzoni ${ }^{3}$ \\ ${ }^{1}$ John Adams Institute at Royal Holloway, University of London, \\ Egham, Surrey TW20 OEX, United Kingdom \\ ${ }^{2}$ CLASSE, Cornell University, Ithaca, New York 14853, USA \\ ${ }^{3}$ CERN, CH-1211 Geneva 23, Switzerland \\ ${ }^{4}$ Cockcroft Institute, Daresbury, Warrington, Cheshire WA4 4AD, United Kingdom \\ ${ }^{5}$ University of Liverpool, Liverpool, Merseyside L69 3BX, United Kingdom
}

(Received 30 October 2014; published 3 April 2015)

\begin{abstract}
Charged particle beam diagnostics is a key task in modern and future accelerator installations. The diagnostic tools are practically the "eyes" of the operators. The precision and resolution of the diagnostic equipment are crucial to define the performance of the accelerator. Transition and diffraction radiation (TR and DR) are widely used for electron beam parameter monitoring. However, the precision and resolution of those devices are determined by how well the production, transport and detection of these radiation types are understood. This paper reports on simulations of TR and DR spatial-spectral characteristics using the physical optics propagation (POP) mode of the Zemax advanced optics simulation software. A good consistency with theory is demonstrated. Also, realistic optical system alignment issues are discussed.
\end{abstract}

DOI: 10.1103/PhysRevSTAB.18.042801

PACS numbers: 41.75.Ht, 41.60.-m

\section{INTRODUCTION}

Beam dynamic considerations demand very tight tolerances on most beam parameters and these in turn dictate most of the requirements for beam instrumentation. In future linear colliders, particle beams with extremely small emittances will be generated in the damping rings [1] and these must be conserved over several tens of $\mathrm{km}$ of beam lines [2]. This requires a precise control of the beam size and emittance over a long distance. In most cases, the measurement of the beam size serves directly to compute the transverse beam emittance. For next generation linear colliders such as the Compact Linear Collider (CLIC [3]) or the International Linear Collider (ILC [2]) and also X-Ray FELs [4], transverse beam size measurements must have a resolution on a micron-scale. A wire scanner does not only have a slightly disturbing effect on the beam, it can also be destroyed by high intensity beams [5]. A laser-wire scanner is the main candidate for noninvasive high resolution measurements [6], but over such long distances many laser-wire monitors would be required which is both costly and difficult to maintain.

Optical transition radiation (OTR) appearing when a charged particle crosses a boundary between two media with different dielectric properties has widely been used as a

\footnotetext{
*Thomas.Aumeyr@rhul.ac.uk

Published by the American Physical Society under the terms of the Creative Commons Attribution 3.0 License. Further distribution of this work must maintain attribution to the author(s) and the published article's title, journal citation, and DOI.
}

tool for transverse profile measurements of charged particle beams in various facilities worldwide [7,8]. The angular distribution is used for beam angular divergence measurements [9]. OTR monitors are simple, robust, and give a direct image of a two-dimensional beam profile. The resolution of the OTR monitors is normally defined as a root-mean-square of the so-called OTR point spread function (PSF) [10]. Recently in [11], the authors have demonstrated that the OTR PSF has a structure which can be used to extract information about the beam size with sub-micrometer resolution [12]. However, OTR based monitors are invasive. They can degrade the beam properties or the beam can destroy the OTR target. Nevertheless, it still can be used to diagnose low intensity "pilot" beams in linear colliders.

Diffraction radiation (DR) is produced when a relativistic charged particle moves in the vicinity of a medium [13]. The spatial-spectral properties of DR are sensitive to a range of electron beam parameters [14-17]. Furthermore, the energy loss due to DR is so small that the electron beam parameters are unchanged. Therefore DR can be used to develop noninvasive diagnostic tools.

In order to study and optimize any optical system, it is essential that real dimensions and optical elements including aberrations are taken into account. The Zemax Optical Design Program is a readily available commercial software package which integrates all the features required to conceptualize, design, optimize, analyze and tolerance virtually any optical system [18]. Zemax uses diffraction calculations to propagate a wavefront through an optical system surface by surface. The wavelike nature of light is fully accounted for. 
Another dedicated software tool dealing with the transport of radiation from electron beams through optical systems and a publication using such software can be found in [19] and [20].

A comparison of the simulation results with state of the art theoretical models was performed and an excellent agreement was achieved when applying the relevant approximations. Furthermore, TR and DR for target and observation properties which go beyond the scope of the existing theory are presented in order to demonstrate the power and flexibility of the developed tool.

\section{ZEMAX}

Zemax supports two modes, geometrical ray tracing and physical optics propagation (POP). Strictly speaking, geometrical ray tracing can only be used when the diffraction effect is negligible. In POP mode, diffraction laws are used to propagate an electric field through an optical system interface by interface, therefore taking into account the full wavelike nature of light.

The wave is modeled using a 2D array of discretely sampled points, analogous to the discrete sampling using rays for geometric optics analysis. Each point in the array stores complex amplitude information about the electric field. The entire array is then propagated in free space between optical interfaces. At each optical interface, a transfer function is computed which propagates the beam from one side of the optical surface to the other, using either Fresnel diffraction propagation or an angular spectrum propagation algorithm [21,22], depending on the highest numerical accuracy. The diffraction propagation algorithms can be used for any propagation distance, for any arbitrary source and can account for any surface aperture, including user defined apertures (UDAs).

Zemax propagates any spatial complex electric field $\boldsymbol{E}$ defined by a $2 \mathrm{D}$ matrix. By keeping track of the electric field components along both the $x$ and $y$ axes, polarization effects can be studied, such as transmission and reflection losses, polarization aberrations and the polarization state of the beam.

Several types of electric fields are already predefined as sources in POP, but any source of light can be provided by the user. The user has to define, among others, the spatial distribution of the complex electric field of the source either in a Beam File or in a DLL [23].

The number of array points describing a beam remains constant as it propagates, but the array size and point spacings will change. A large array width at the beam waist compared to its waist size results in only a few points across the beam waist and thus a smaller array size far from the waist with more points across beam size. On the other hand, with a smaller array at the waist the array will grow large compared to the beam far from the waist resulting in only a few sample points across beam. Therefore, there is a trade-off between good sampling of the beam near the waist and good sampling far from the waist [24].

\section{TRANSITION RADIATION}

\section{A. Model}

Transition radiation (TR) appears when a charged particle crosses a boundary between two media with different dielectric properties. The theory used in this paper is based on Huygens principle of wave diffraction [25], i.e., when the incident particle field is introduced as a superposition of so-called pseudo-photons which reflect off the interface and propagate through an optical system.

For the purpose of this paper only the vertical polarization component of TR is considered. This is a viable approach given the rotational symmetry of the simplest TR case and the asymmetric cases being studied introducing such asymmetries only in the vertical plane. The approximation of $y$ polarization component of the OTR electric field induced by a single electron on a target surface, is used as the source in the simulations. This is based on the solution of Maxwell equations for a field of a charged particle moving in free space. Its real and imaginary parts, $\Re\left\{E_{y}\right\}$ and $\Im\left\{E_{y}\right\}$, are represented as [26]

$$
\begin{aligned}
\Re\left\{E_{y}\right\}= & \frac{2 e}{\gamma \lambda \cos \theta_{0}}\left\{\frac{y}{\rho} K_{1}\left[\frac{2 \pi}{\beta \gamma \lambda} \rho\right] \cos \phi \cos \theta_{0}\right. \\
& \left.+\frac{1}{\gamma} K_{0}\left[\frac{2 \pi}{\beta \gamma \lambda} \rho\right] \sin \phi \sin \theta_{0}\right\} \\
\Im\left\{E_{y}\right\}= & \frac{2 e}{\gamma \lambda \cos \theta_{0}}\left\{\frac{y}{\rho} K_{1}\left[\frac{2 \pi}{\beta \gamma \lambda} \rho\right] \sin \phi \cos \theta_{0}\right. \\
& \left.-\frac{1}{\gamma} K_{0}\left[\frac{2 \pi}{\beta \gamma \lambda} \rho\right] \cos \phi \sin \theta_{0}\right\},
\end{aligned}
$$

with $\beta=\sqrt{1-\frac{1}{\gamma^{2}}}$, phase shift $\phi=k y \tan \theta_{0}\left(1-\frac{1}{\beta}\right), k=\frac{2 \pi}{\lambda}$ and $\rho=\sqrt{x^{2}+y^{2}}$. Here $x$ and $y$ are two orthogonal coordinates of the target measured from the point of electron incidence, $\gamma$ is the charged particle Lorentz factor, $\lambda$ is the radiation wavelength, $\theta_{0}$ is the angle between the particle trajectory normal and the target plane, $K_{0}$ and $K_{1}$ are the zeroth and first order modified Bessel function. For TR, the entire field is propagated toward the observation plane.

This field used as the initial input for the simulation is essentially the field at the target surface projected onto the $x-y$ plane. This approximation is valid for distances much larger than $\frac{\gamma \lambda}{2 \pi}$, which in all simulations presented in this paper is of the order of $10^{-4} \mathrm{~m}$ or less. The geometry is illustrated in Fig. 1 for further clarification. The $x$-axis is always in the plane of the target. The $z$-axis is along the mirror reflection direction. The incident electron trajectory is in the $y-z$ plane. 


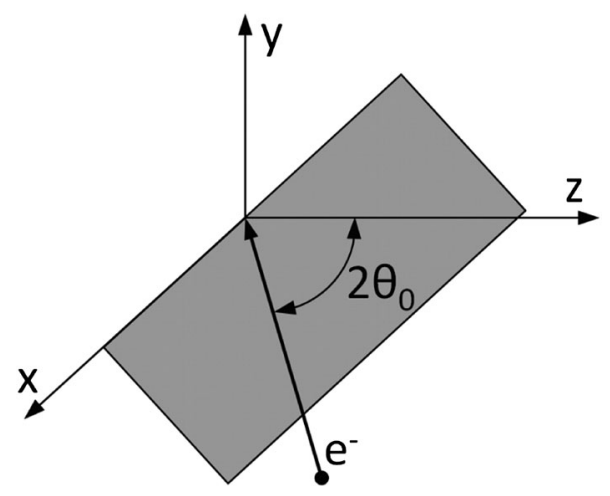

FIG. 1. Geometry of the source field generation.

The irradiance at the image plane, $I$, can be calculated using

$$
I=\Re\left\{E_{y}\right\}^{2}+\Im\left\{E_{y}\right\}^{2} .
$$

Following the approach for calculating TR from a particle passing through a boundary between vacuum and an ideal conductor [7] and applying an ultrarelativistic approximation $\left(\theta_{x}, \theta_{y}, \gamma^{-1} \ll 1\right)$, the following equation is obtained for the vertically polarized component of the spectral-angular distribution of intensity [27]:

$$
\frac{d^{2} W_{y}^{T R}}{d \omega d \Omega}=\frac{\alpha}{\pi^{2}} \frac{\theta_{y}{ }^{2}}{\left(\gamma^{-2}+\theta_{x}{ }^{2}+\theta_{y}{ }^{2}\right)^{2}} \frac{1}{\left(1-\theta_{y} \tan \theta_{0}\right)^{2}} .
$$

Here $\theta_{x}$ and $\theta_{y}$ are the radiation observation angles measured either from the mirror reflection direction or from the particle trajectory and $\alpha$ is the fine structure constant. It can be seen that for $\theta_{y}=\frac{1}{\gamma}$ and $\theta_{x}=\theta_{0}=0$, the intensity is maximum with a value of $\frac{\alpha \gamma^{2}}{4 \pi^{2}}$. All simulations presented in this paper are normalized with respect to this TR maximum intensity.

\section{B. Propagation in free space}

In order to demonstrate the validity of the simulation method, this sections deals with far-field simulations and compares the results with well-known analytical theory. The simulation parameters for each figure were chosen to highlight certain characteristics of TR and are stated in the caption accordingly. To assume far-field observation geometry, the propagation distance in free space between the target and observation planes must satisfy the condition $L \gg \frac{\gamma^{2} \lambda}{2 \pi}$, i.e., $L$ must be much larger than so-called radiation formation length [10]. This is the far-field requirement for TR.

Figure 2 shows the effect on the angular distribution when moving the detector plane from the pre-wave zone into the far-field. The size of the source was $r_{\max }=10 \frac{\gamma \lambda}{2 \pi}$ to fulfil an infinite target approximation, i.e., ten times the

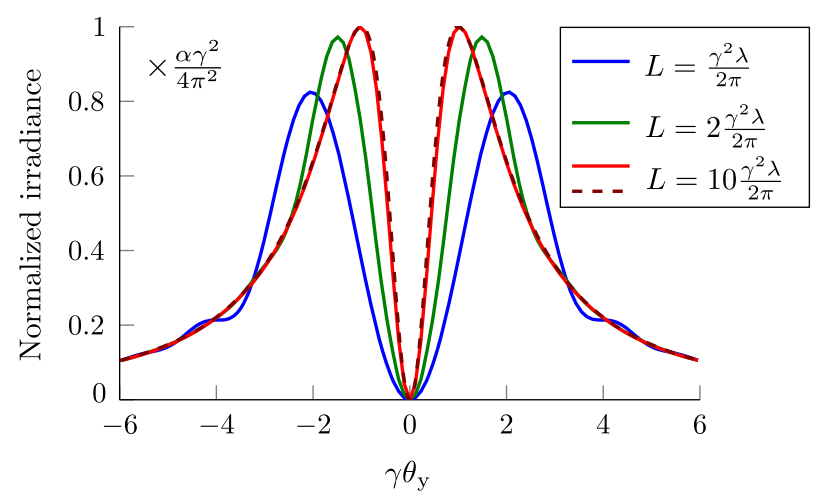

FIG. 2. Comparison between theoretical (dashed) and simulated (solid) TR angular distribution for various distances between source and image plane $L\left(\lambda=550 \mathrm{~nm}, \gamma=2500, \theta_{x}=0\right)$.

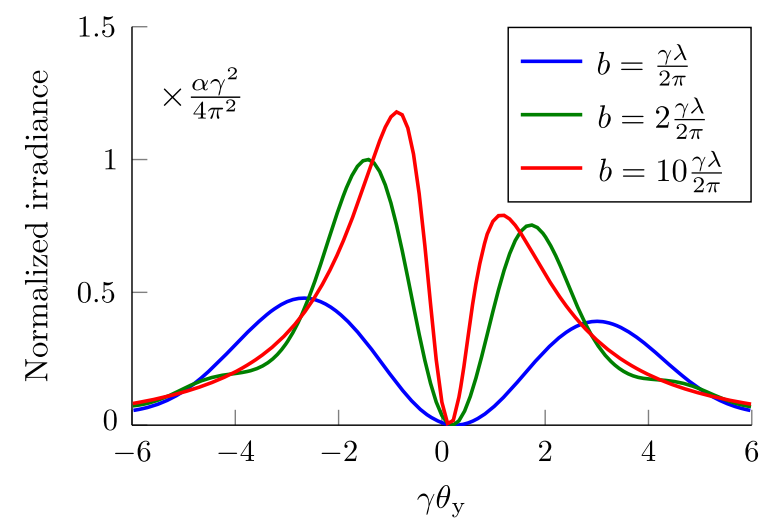

FIG. 3. TR angular distribution in the far-field using a tilted screen and for various target disc radii $b(\lambda=550 \mathrm{~nm}, \gamma=10$, $\theta_{x}=0, \theta_{0}=45^{\circ}$.

radius of the electric field. This figure is in excellent agreement with analytical calculations presented in [28].

In Fig. 3, the TR angular distribution in the far-field is shown when using a screen tilted at an angle of $\theta_{0}=45^{\circ}$ and for various target dimensions. For the first time, it combines effects of the target tilt and finite outer dimensions which is a very complicated task to be realized analytically and thus shows the power of the simulation tool. This plot was done for a small Lorentz factor of $\gamma=10$ to highlight the asymmetry of the pattern for lower beam energies.

It can be seen that for lower beam energies and large target dimensions there is a strong asymmetry in the TR angular distribution. For smaller dimensions of the target however, the asymmetry reduces and the intensity decreases as well.

\section{PSF analysis for a real optical system}

To study the PSF, the simulated detector plane has to be in the image plane of a lens (Fig. 4). 


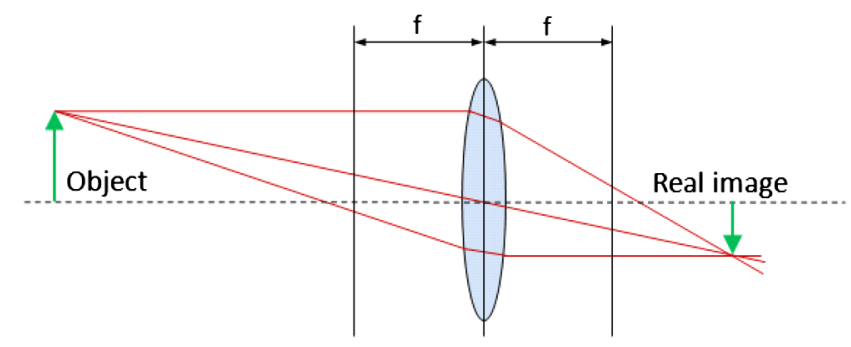

FIG. 4. Setup for studying the PSF.

The power of the ZEMAX tool is that any optical system can be built and nominally adjusted to a real one by using exact commercial products. In the case of the OTR monitor at the Accelerator Test Facility (ATF2) [14], the lens is an achromatic Sigma Koki DLB-30-120PM with a focal length of $f=120.1 \mathrm{~mm}$, set up with a magnification of $M=-7.4$. The PSF is highly sensitive to misalignment, which is presented in Fig. 5a. The asymmetry of the TR PSF can be expressed by taking the peak irradiance of the left and right main lobes, $I_{L}$ and $I_{R}$, and compute $\left(I_{L}-I_{R}\right) /\left(I_{L}+I_{R}\right)$. In Fig. $5 \mathrm{~b}$, it can be seen that the asymmetry is growing when increasing the horizontal lens offset (transverse to the optical axis).

Displacing the lens longitudinally from the image plane causes the TR PSF to broaden, as can be observed in Fig. 6a. Plotting the distance between the two main lobes of the TR PSF for different offsets shows that minimum peak separation is achieved at the image plane (see Fig. 6b).

In Fig. $7 \mathrm{a}$ and $7 \mathrm{~b}$, the effects of diffraction with a too small lens are demonstrated. Using a biconvex lens with a
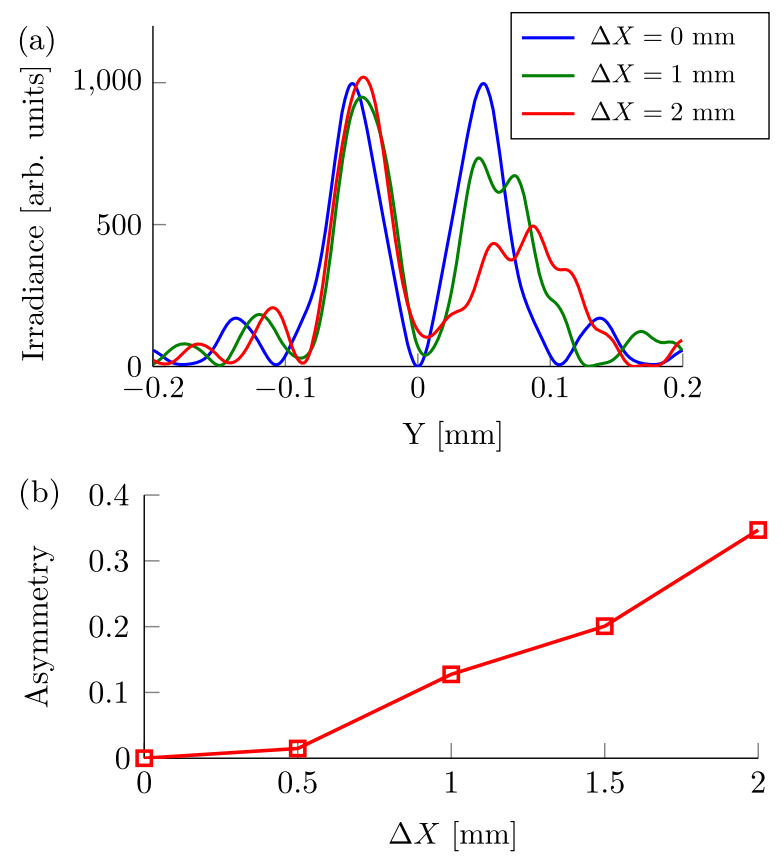

FIG. 5. TR PSF (a) and asymmetry (b) for various horizontal lens offsets $\Delta X\left(\lambda=550 \mathrm{~nm}, \gamma=2500, \theta_{x}=0, M=-7.4\right)$.
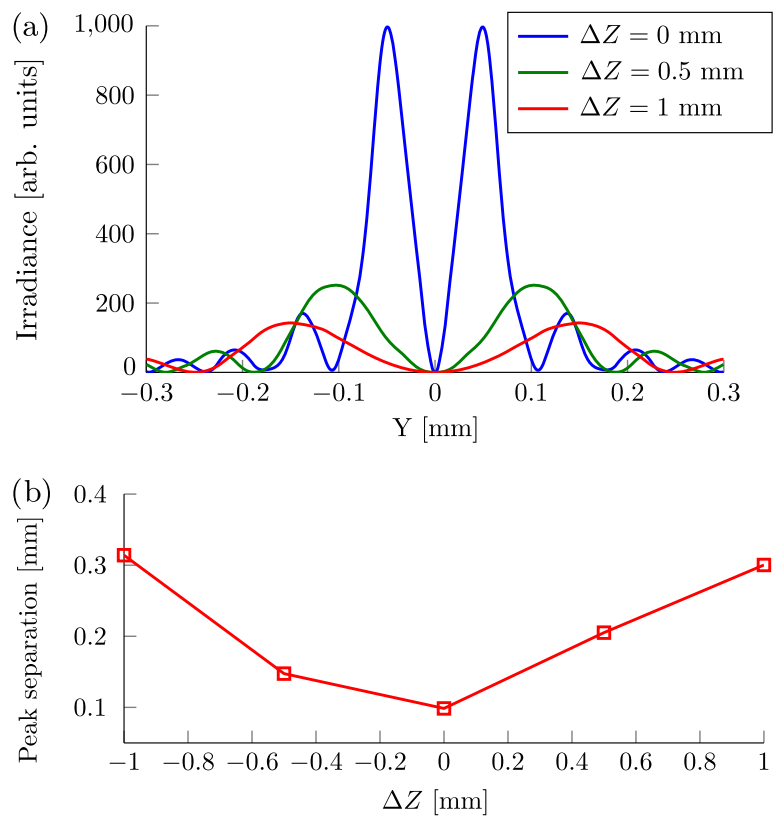

FIG. 6. TR PSF (a) and distance between the two main lobes (b) for various longitudinal lens offsets $\Delta Z(\lambda=550 \mathrm{~nm}, \gamma=2500$, $\left.\theta_{x}=0, M=-7.4\right)$.

focal length of $f=100$, set up with a magnification of $M=-10$, the distance between the two main lobes of the TR PSF is measured while changing the lens diameter. It can be seen that diffraction is reduced when increasing the size of the lens, thus decreasing the peak separation until the introduction of aberrations increases the peak distance
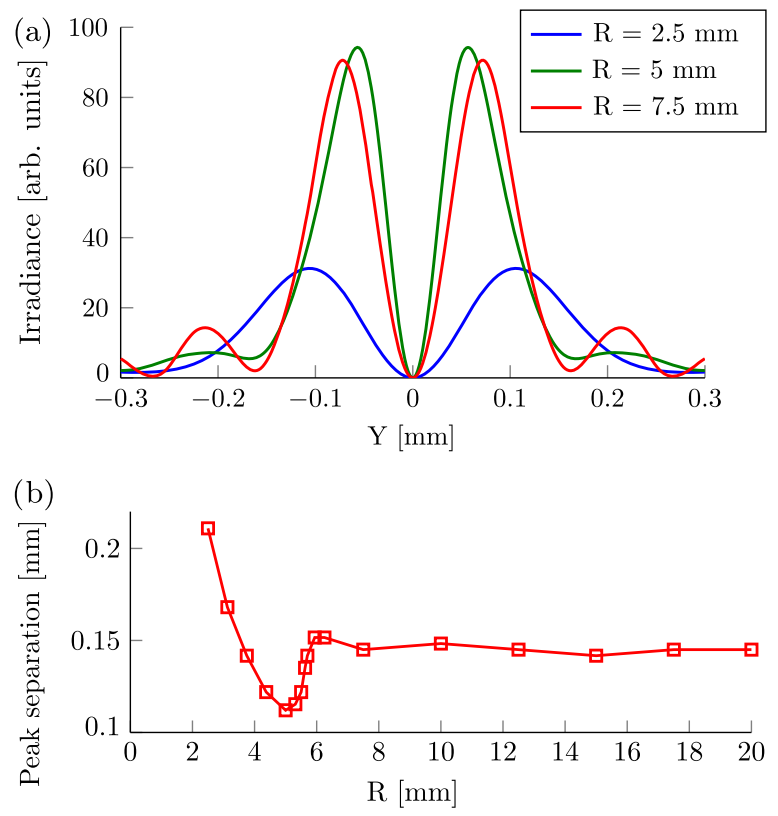

FIG. 7. TR PSF (a) and distance between the two main lobes (b) for various lens radii $R\left(\lambda=550 \mathrm{~nm}, \gamma=2500, \theta_{x}=0, M=-10\right)$. 
again. The peak separation remains constant as soon as the entire OTR light is captured by the lens.

\section{DIFFRACTION RADIATION}

Diffraction radiation (DR) is produced when a relativistic charged particle passes through an aperture or near a discontinuity in the media in which it is traveling.

\section{A. Diffraction radiation from a circular aperture in an opaque screen}

The first models appeared over 60 years ago [29]. The model used for comparison in this section is presented in [13]. For a charged particle moving normally through the center of a circular hole in an infinitely thin, perfectly conducting disc, the following analytical expression for the electric field in the far-field can be derived

$$
E_{y}^{\mathrm{hole}}(\mathbf{r}, \omega)=\frac{i e}{\pi \gamma} \frac{F(b)-F(a)}{\gamma^{-2}+\theta_{x}^{2}+\theta_{y}^{2}}
$$

using

$F(a)=a k \theta J_{0}(a k \theta) K_{1}\left(\frac{a \omega}{v \gamma}\right)+\frac{a \omega}{v \gamma} J_{1}(a k \theta) K_{0}\left(\frac{a \omega}{v \gamma}\right)$.

The spectral-angular distribution can be calculated as

$$
\frac{d^{2} W_{y}^{\text {hole }}}{d \omega d \Omega}=4 \pi^{2}\left|E_{y}^{\text {hole }}\right|^{2}
$$

Figure 8 shows DR angular distributions in the far-field for target discs of various radii and a hole radius of $a=1 \mathrm{~nm}$, i.e., assuming an infinitely small hole.

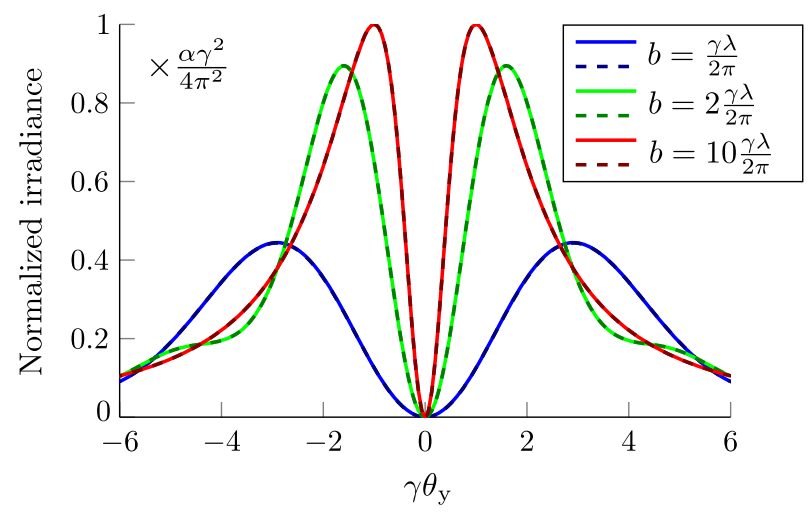

FIG. 8. Comparison between theoretical (dashed) and simulated (solid) DR angular distribution in the far-field for various target disc radii $b\left(\lambda=400 \mathrm{~nm}, \gamma=4110, \theta_{x}=0, a=1 \mathrm{~nm}\right)$.

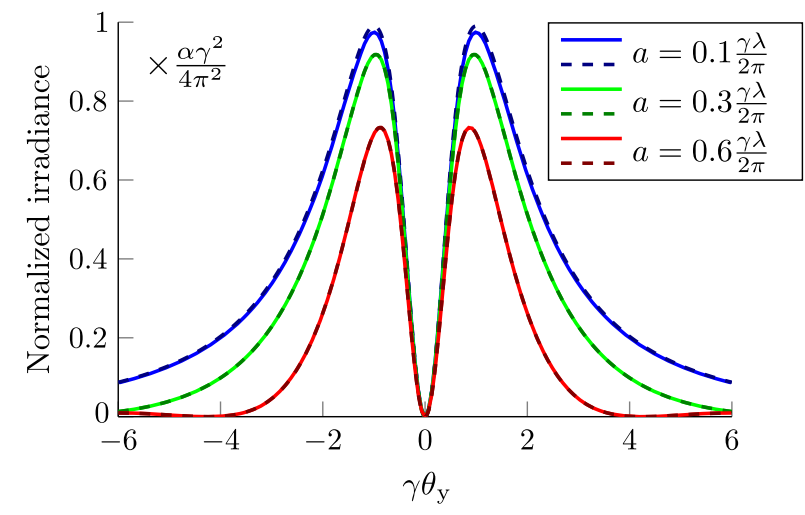

FIG. 9. Comparison between theoretical (dashed) and simulated (solid) DR angular distribution in the far-field for various hole radii $a\left(\lambda=400 \mathrm{~nm}, \gamma=4110, \theta_{x}=0, b=10 \frac{\gamma \lambda}{2 \pi}\right)$.

In Fig. 9, the disc radius is kept constant as $b=10 \frac{\gamma \lambda}{2 \pi}$ and the hole radius is varied. For both cases, a good agreement between theory and simulation has been achieved [13,29].

In Fig. 10, the angular distribution in the far-field for DR coming from a circular hole with a radius of $a=\frac{\gamma \lambda}{2 \pi}$ for various tilt angles is shown. Here, a Lorentz factor of $\gamma=10$ was used to showcase the asymmetry of the pattern for low energies. The plot highlights the increasing asymmetry of the angular distribution for large tilt angles. The increase in overall intensity simply results from the decrease in effective hole diameter in the projection onto the source plane, which is perpendicular to the propagation of the electron beam.

In [7], the flight of an electron through a circular hole is calculated for finite offsets from the center of the hole. However, the applied limitations over approximate the analytical expression, as it is only valid for very small holes $\left(a \ll \frac{\gamma \lambda}{2 \pi}\right)$ and even smaller offsets $(r \ll a)$. Therefore, simulations have to be used to study this case.

In Fig. 11, the irradiance at the source for a single electron passing through a circular hole can be seen. This setup shows an offset of the particle with respect to the hole

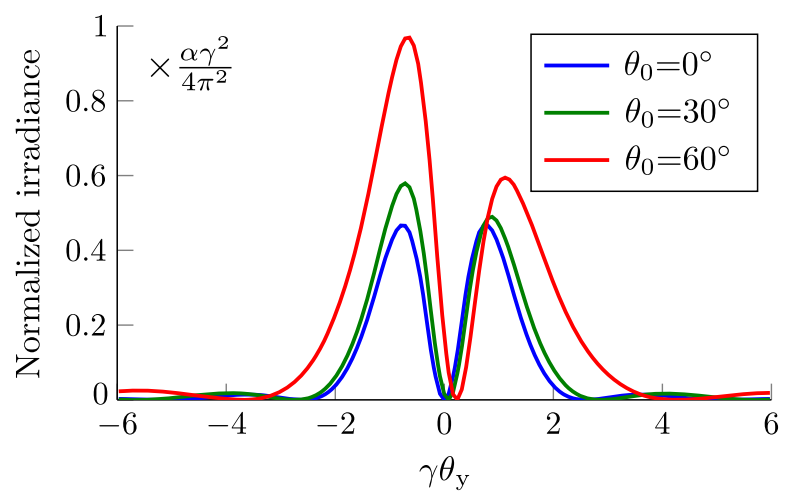

FIG. 10. DR angular distribution in the far-field for various tilt angles $\theta_{0}\left(\lambda=400 \mathrm{~nm}, \gamma=10, \theta_{x}=0, a=\frac{\gamma \lambda}{2 \pi}, b=10 \frac{\gamma \lambda}{2 \pi}\right)$. 


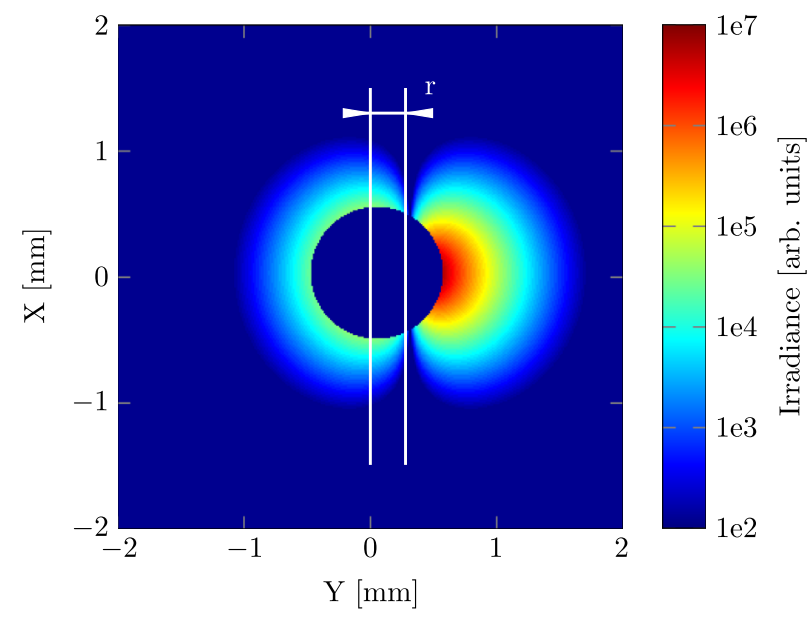

FIG. 11. Vertically polarized irradiance at the source for single electron passing through circular hole with a beam offset of $r=\frac{\gamma \lambda}{2 \pi}\left(\lambda=400 \mathrm{~nm}, \gamma=4110, a=2 \frac{\gamma \lambda}{2 \pi}\right)$.

center. The field at the source is then propagated using Zemax. Figure 12 shows a 2D contour plot of angular distribution versus particle position with respect to the center of the hole $r$ with a radius of $a=2 \frac{\gamma \lambda}{2 \pi}$ in the far-field. Each horizontal line represents the cross-section of the angular distribution for a beam of a certain offset. Starting with a beam offset of $r=-4 \frac{\gamma \lambda}{2 \pi}$, the distribution is almost pure TR. The closer the beam moves up to the center of the hole, the more DR-like the distribution becomes. It can be seen that in the far-field, the transition from TR to DR shows the expected symmetry.

In the pre-wave zone, i.e., for a distance of $5 \frac{\gamma^{2} \lambda}{2 \pi}$ between the source and the observation plane, an asymmetry can be

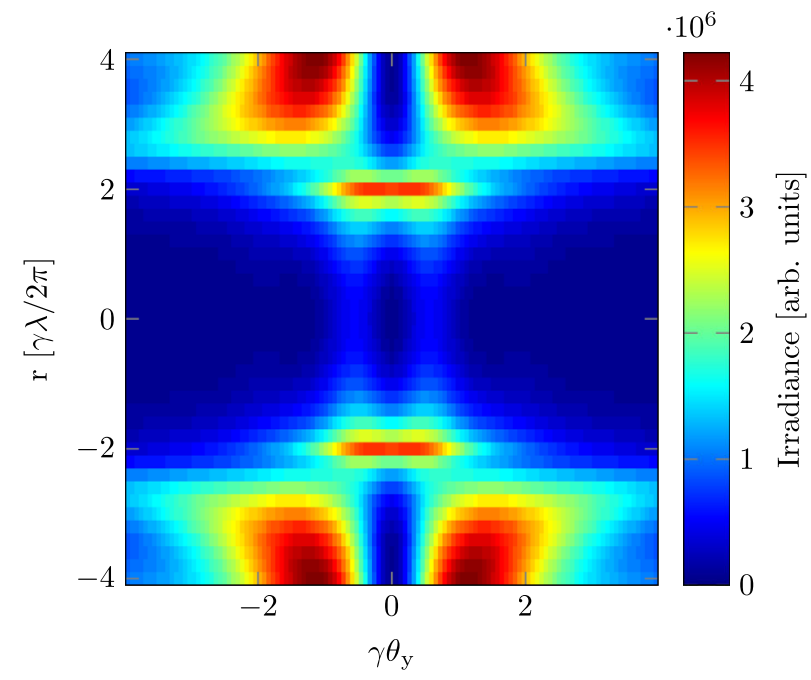

FIG. 12. DR angular distribution in the far-field for various beam offsets $r\left(\lambda=400 \mathrm{~nm}, \gamma=4110, a=2 \frac{\gamma \lambda}{2 \pi}, L=100 \frac{\gamma^{2} \lambda}{2 \pi}\right)$.

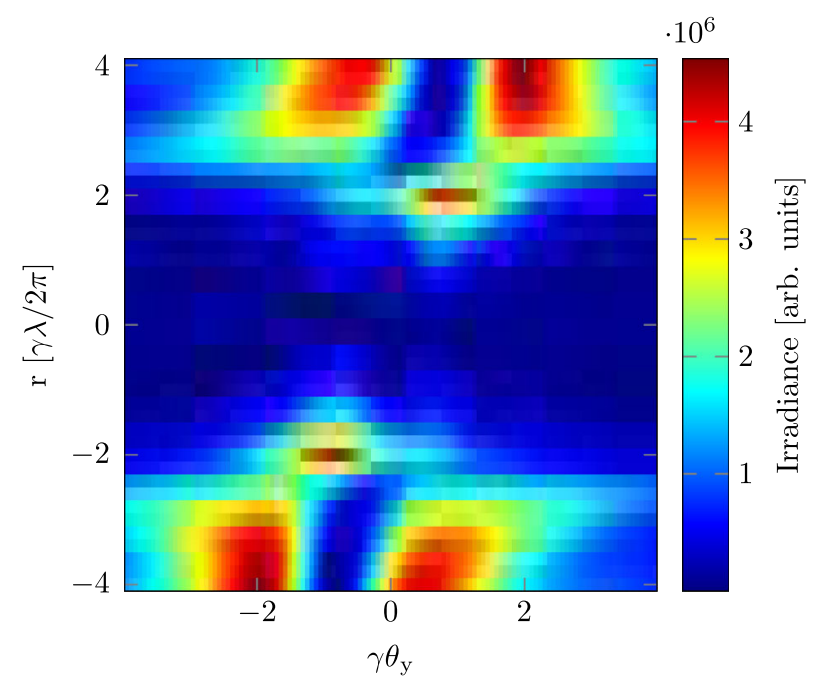

FIG. 13. DR angular distribution in the pre-wave zone for various beam offsets $r\left(\lambda=400 \mathrm{~nm}, \gamma=4110, a=2 \frac{\gamma \lambda}{2 \pi}, L=5 \frac{\gamma^{2} \lambda}{2 \pi}\right)$.

found (see Fig. 13). This originates from the interference of the radiation from different parts of the target.

\section{B. Diffraction radiation from a slit aperture in an opaque screen}

This model considers the case, when a charged particle moves through a slit between two semi-planes, i.e., only DR produced from the target is considered. In the case of a horizontal slit, the vertical polarization component is sensitive to beam size [14]. Equation (8) gives the expression for the vertical polarization component of optical diffraction radiation (ODR) convoluted with a Gaussian distribution [14], where $t_{x, y}=\gamma \theta_{x, y}, \lambda$ is the observation wavelength, $\sigma_{y}$ is the rms vertical beam size, $a$ is the effective target aperture size (the real one is $a=a / \cos \theta_{0}$, where $\theta_{0}$ is the angle between the particle trajectory normal and the target), $\overline{a_{x}}$ is the offset of the beam center with respect to the center of the slit and $\psi=\arctan \left(t_{y} / \sqrt{1+t_{x}^{2}}\right)$. This model is applicable when the transition radiation contribution from the tails of the Gaussian distribution is negligible, which means approximately $a \geq 4 \sigma_{y}$.

$$
\begin{aligned}
\frac{d^{2} W_{y}^{\text {slit }}}{d \omega d \Omega}= & \frac{\alpha \gamma^{2}}{2 \pi^{2}} \frac{\exp \left(-\frac{2 \pi a}{\gamma \lambda} \sqrt{1+t_{x}^{2}}\right)}{1+t_{x}^{2}+t_{y}^{2}} \\
& \times\left\{\exp \left[\frac{8 \pi^{2} \sigma_{y}^{2}}{\lambda^{2} \gamma^{2}}\left(1+t_{x}^{2}\right)\right] \cosh \left(\frac{4 \pi \overline{a_{x}}}{\gamma \lambda} \sqrt{1+t_{x}^{2}}\right)\right. \\
& \left.-\cos \left(\frac{2 \pi a}{\gamma \lambda} t_{y}+2 \psi\right)\right\} .
\end{aligned}
$$

This expression is valid for ultra-relativistic particles and small target tilt angles. For a slit width $a=0$ follows 


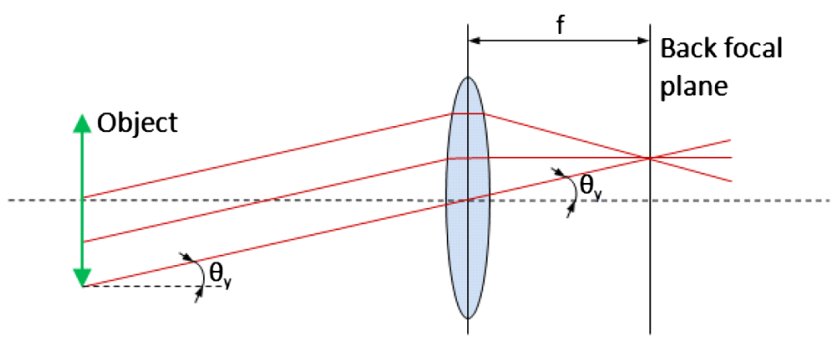

FIG. 14. Setup for studying the angular distribution in the prewave zone.

considering the above approximation that $\overline{a_{x}}=0$ and $\sigma_{y}=0$, that Eq. (8) results in the angular distribution for OTR in Eq. (4).

To obtain the DR angular distribution in the pre-wave zone, a focusing lens with the detector positioned in the back focal plane is used to remove the spatial contribution (see Fig. 14).

Figure 15 compares the distribution at the detector plane for three different setups: far-field, pre-wave zone using an ideal paraxial lens with a focal length of $f=500 \mathrm{~mm}$ and pre-wave zone with a plano-convex Thorlabs LA4782-UV lens $(f=500 \mathrm{~mm}, \oslash 50.8 \mathrm{~mm})$, as used in the real optical setup at CesrTA [30]. The results of all three setups are in excellent agreement.

All kinds of offsets, tilts and other misalignments of real optical systems can be studied. To illustrate the power of this method, Fig. 16 shows the DR angular distribution for various vertical lens tilts. It can be seen that a very serious distortion of the ODR angular distribution occurs at large lens tilt angles. However, an angular lens alignment with accuracy much better than $10^{\circ}$ can easily be achieved.

For completeness, Fig. 17 shows the DR angular distribution for various vertical lens offsets. In this case though, barely any distortion in the distribution pattern can be observed, even for large lens offsets.

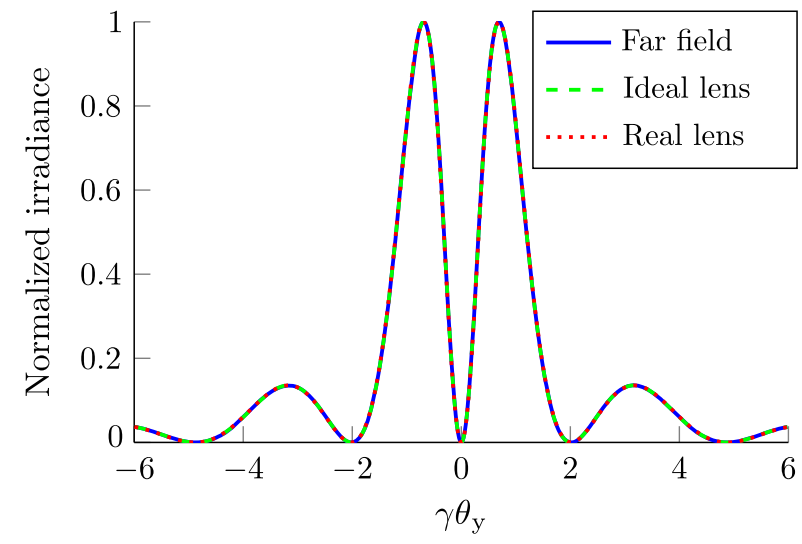

FIG. 15. Comparison of the DR angular distribution in the far-field, in the back focal plane of an ideal lens and in the back focal plane of the plano-convex lens $\left(\lambda=400 \mathrm{~nm}, \gamma=4110, \theta_{x}=0, a=\frac{\gamma \lambda}{2 \pi}\right)$.

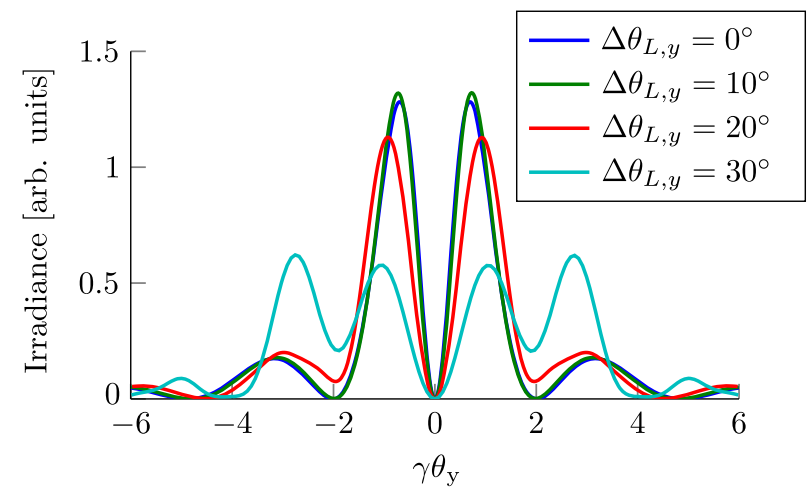

FIG. 16. DR angular distribution in the back focal plane of the plano-convex lens for various vertical lens tilts $\Delta \theta_{L, y}$ $\left(\lambda=400 \mathrm{~nm}, \gamma=4110, \theta_{x}=0, a=\frac{\gamma \lambda}{2 \pi}\right)$.

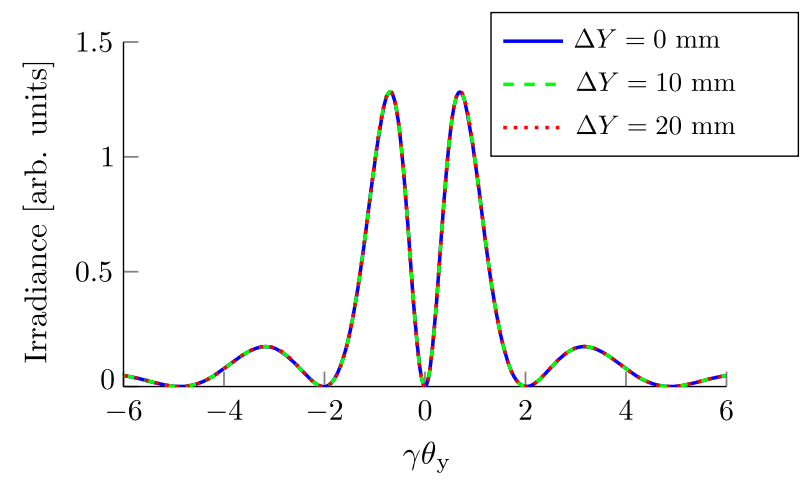

FIG. 17. DR angular distribution in the back focal plane of the plano-convex lens for various vertical lens offsets $\Delta Y$ $\left(\lambda=400 \mathrm{~nm}, \gamma=4110, \theta_{x}=0, a=\frac{\gamma \lambda}{2 \pi}\right)$.

\section{Diffraction radiation for an arbitrarily shaped aperture}

Zemax can also be used to simulate the vertical polarization component of the DR angular distribution using a

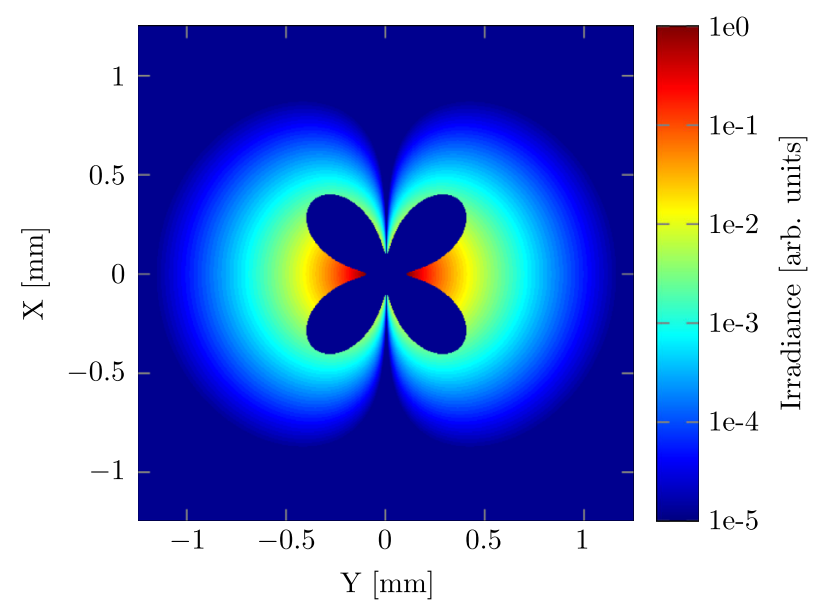

FIG. 18. Vertically polarized irradiance at the source for a fourleafed aperture $\left(\lambda=400 \mathrm{~nm}, \gamma=4110, a=2 \frac{\gamma \lambda}{2 \pi}\right)$. 


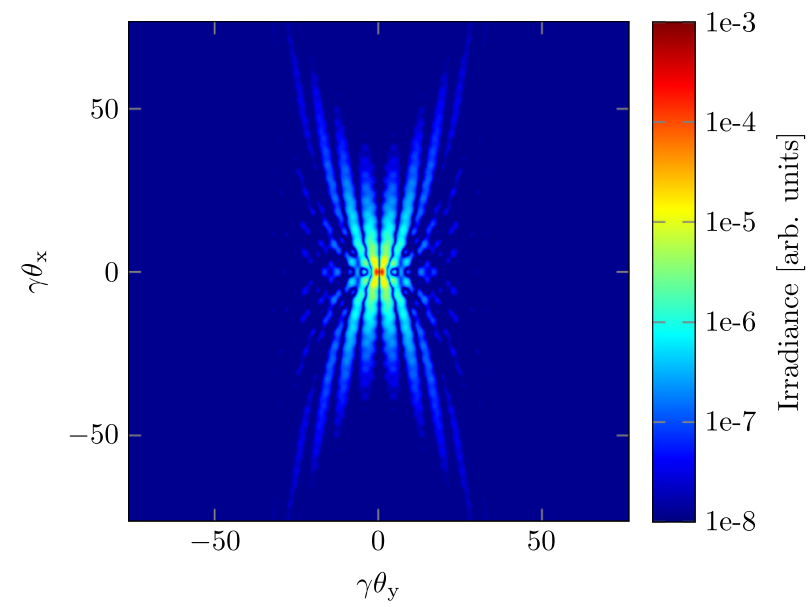

FIG. 19. DR angular distribution vertical polarization for a four-leafed aperture in the far-field $(\lambda=400 \mathrm{~nm}, \gamma=4110$, $\left.a=2 \frac{\gamma \lambda}{2 \pi}, L=10 \frac{\gamma^{2} \lambda}{2 \pi}\right)$.

target with arbitrarily shaped aperture. As an example, a collimator with a four-leafed aperture can be described as

$$
\left(x^{2}+y^{2}\right)^{3}=4 a^{2} x^{2} y^{2},
$$

where $a$ is the size of the leaf. The source distribution with a leaf size of $a=2 \frac{\gamma \lambda}{2 \pi}$ is shown in Fig. 18, the corresponding angular distribution in the far-field can be seen in Fig. 19. Both plots shown are total irradiance plots displayed in a logarithmic scale.

\section{CONCLUSIONS}

In this paper, advanced Zemax simulations of OTR and ODR were presented and compared with state-ofthe-art theoretical models. Misalignment studies were carried out to study the limitations of real optical systems. Furthermore, arbitrary ODR targets were simulated to highlight the power of this method.

With assumptions similar to theoretical boundary conditions, Zemax simulations of TR and DR agree with the analytical expressions. The simulations even include a tilt angle in the incident electric fields to enable simulating a tilted target. Introducing an off-axis incident field or even an arbitrarily shaped aperture does not slow down the Zemax simulations noticeably and is therefore the preferable method. These simulations also enable applying a finite beam size. This can be achieved by displacing the single particle with respect to the optical axis across the transversal profile. The resulting angular pattern for each step can then be weighted and summed up.

Overall, this tool represents the most comprehensive approach to the design of a real diagnostics based on either OTR or ODR including all misalignment errors and optimization of a real optical system (including viewports, polarizers, filters, etc.). Field depth studies can then be done as well as investigations on the behavior of the TR PSF in real optical setups.

\section{ACKNOWLEDGMENTS}

The research leading to these results has received funding from the Science and Technology Facilities Council via the John Adams Institute at Royal Holloway, University of London. We would like to acknowledge CERN for financial support of this research within the CLIC-UK collaboration: Contract No. KE1870/ DG/CLIC. This research was also partially supported by the oPAC: FP7 Marie Curie Initial Training Network under Contract No. PITN-GA-2011-289485.

[1] G. R. White et al. (ATF2 Collaboration), Phys. Rev. Lett. 112, 034802 (2014).

[2] T. Behnke, J. E. Brau, B. Foster, J. Fuster, M. Harrison et al., The International Linear Collider Technical Design Report Volume 1: Executive Summary (2013).

[3] H. Schmickler et al., CLIC Conceptual Design Report No. CERN-2012-07, 2012.

[4] R. Abela et al., DESY XFEL: The European X-Ray Free-Electron Laser - Technical Design Reports No. PHPPUBDB-14956 and DESY 06-097, 2006.

[5] K. Kubo et al. (ATF Collaboration), Phys. Rev. Lett. 88, 194801 (2002).

[6] L. J. Nevay, R. Walczak, and L. Corner, Phys. Rev. ST Accel. Beams 17, 072801 (2014).

[7] M. L. Ter-Mikaelian, High Energy Electromagnetic Processes in Condensed Media (Wiley, New York, 1972).

[8] C. Thomas, N. Delerue, and R. Bartolini, J. Instrum. 6, P07004 (2011).

[9] R. Fiorito and D. Rule, Nucl. Instrum. Methods Phys. Res., Sect. B 173, 67 (2001).

[10] M. Castellano and V. A. Verzilov, Phys. Rev. ST Accel. Beams 1, 062801 (1998).

[11] P. Karataev, A. Aryshev, S. Boogert, D. Howell, N. Terunuma, and J. Urakawa, Phys. Rev. Lett. 107, 174801 (2011).

[12] M. Ross, S. Anderson, J. Frisch, K. Jobe, D. McCormick, B. McKee, J. Nelson, T. Smith, H. Hayano, T. Naito et al., Report No. SLAC-PUB-9280, 2002.

[13] A. P. Potylitsyn, M. I. Ryazanov, M. N. Strikhanov, and A. A. Tishchenko, Springer Tracts Mod. Phys. 239, 1 (2011).

[14] P. Karataev, S. Araki, R. Hamatsu, H. Hayano, T. Muto, G. Naumenko, A. Potylitsyn, N. Terunuma, and J. Urakawa, Phys. Rev. Lett. 93, 244802 (2004).

[15] M. Castellano, V. A. Verzilov, L. Catani, A. Cianchi, G. Orlandi, and M. Geitz, Phys. Rev. E 63, 056501 (2001).

[16] M. Castellano, Nucl. Instrum. Methods Phys. Res., Sect. A 394, 275 (1997).

[17] J. Urakawa, H. Hayano, K. Kubo, S. Kuroda, N. Terunuma et al., Nucl. Instrum. Methods Phys. Res., Sect. A 472, 309 (2001).

[18] Zemax 13, Optical Design Program, User's Manual (2013). 
[19] B. Schmidt, THzTransport, www.desy.de/schmidtb/ THzTransport/THzTransport.m.

[20] S. Casalbuoni, B. Schmidt, P. Schmüser, V. Arsov, and S. Wesch, Phys. Rev. ST Accel. Beams 12, 030705 (2009).

[21] J. W. Goodman, Introduction to Fourier Optics (McGrawHill, New York, 1968).

[22] G. N. Lawrence, in Optical Modeling, Applied Optics and Optical Engineering Vol. 11, edited by R. R. Shannon and J. C. Wyant (Academic, New York, 1992).

[23] T. Aumeyr, M. G. Billing, L. M. Bobb, B. Bolzon, P. Karataev, T. Lefevre, and S. Mazzoni, JPCS 517, 012026 (2014).
[24] B. Bolzon, CERN Report, 2013.

[25] M. Castellano, A. Cianchi, G. Orlandi, and V. A. Verzilov, Nucl. Instrum. Methods Phys. Res., Sect. A 435, 297 (1999).

[26] D. V. Karlovets and A.P. Potylitsyn, Nucl. Instrum. Methods Phys. Res., Sect. B 266, 3738 (2008).

[27] A. P. Potylitsyn, Nucl. Instrum. Methods Phys. Res., Sect. B 145, 169 (1998).

[28] P. V. Karataev, Phys. Lett. A 345, 428 (2005).

[29] Y. N. Dnestrovskiy and D. P. Kostomarov, Sov. Phys. Dokl. 4, 158 (1959).

[30] D. Rubin, D. Sagan, and J. Shanks, in Proceedings of the 23rd Particle Accelerator Conference, Vancouver, Canada, 2009 (IEEE, Piscataway, NJ, 2009). 